\title{
Indentations and Starting Points in Traveling Sales Tour Problems: Implications for Theory
}

\author{
James N. MacGregor ${ }^{1}$
}

\begin{abstract}
A complete, non-trivial, traveling sales tour problem contains at least one "indentation", where nodes in the interior of the point set are connected between two adjacent nodes on the boundary. Early research reported that human tours exhibited fewer such indentations than expected. A subsequent explanation proposed that this was because the observed human tours were close to the optimal, and the optimal tours happened to have few indentations. The present article reports two experiments. The first was designed to test the "few indentations" hypothesis under more stringent conditions than previously, by including point sets with two (near) optimal solutions that had a different number of indentations. For these critical point sets, participants produced the optimal solution with fewer indentations significantly more often than the alternative optimal solution. In addition, participants' solutions started on boundary points significantly more often than by chance. A second experiment tested whether the preference for fewer indentations is the result of a conscious strategy, or the product of the processes that generate a solution. The results supported the latter conclusion. The implications for theories of human tour generation are discussed.
\end{abstract}

\section{Keywords}

problem solving, optimization, convexity, perception of shorter paths

\footnotetext{
${ }^{1}$ University of Victoria. Please direct correspondence to jmacgreg@uvic.ca.
} 


\section{Introduction}

Combinatorial optimization problems require searching for an optimal solution in a problem space where, typically, the number of possible solutions increases exponentially with the number of problem elements. A common example is the Traveling Salesman Problem (TSP) which, for the two dimensional case, requires finding the shortest tour through a set of points in the plane, returning to the start point. The problem appears in different guises in many industrial and scientific applications and, in its general form, has proven to be computationally intractable. Because of this, computer scientists and operations researchers have focused on finding approximating algorithms that can produce good solutions fairly quickly. Effective approximating algorithms can find solutions that are only a few percent longer than the optimal solution in a time that is a low-order polynomial function of the number of nodes (Pizlo, Stefanov, Saalweachter, Haxhimusa, \& Kropatsch, 2006).

In recent years cognitive scientists have begun to examine human performance in optimization tasks and, although still in its infancy, this new research area has attracted the attention of researchers working in vision (Findlay \& Brown, 2006), memory (Kong, Schunn, \& Wallstrom, 2010), decision making (Ragni \& Löffler, 2010), clinical neuroscience (Basso et al., 2006) and comparative psychology (Gibson, Wasserman, \& Kamil, 2007). Preliminary findings reported in the psychological literature indicated that untrained human subjects can quickly produce optimal or near-optimal solutions to TSPs, and often outperform simple approximating algorithms, such as the nearest neighbour procedure (MacGregor \& Ormerod, 1996). These basic findings have since been confirmed by subsequent research (Graham, Joshi, \& Pizlo, 2000; Vickers, Butavicius, Lee, \& Medvedev, 2001). In addition, human solutions rarely self-intersect (MacGregor, Ormerod, \& Chronicle, 2000; van Rooij, Stege, \& Schactman, 2003; Vickers, Lee, Dry, \& Hughes, 2003) and are produced in times that appear to increase linearly with problem size, rather than polynomially (Dry, Lee, Vickers, \& Hughes, 2006; Pizlo et al., 2006).

While many of the early findings have been corroborated by subsequent research, there are some that have been virtually overlooked. One is the observation that human solutions tend to have fewer "indentations" than might be expected (MacGregor \& Ormerod, 1996). In a set of points in the plane, an indentation occurs each time a point on the boundary of the set (the "convex hull") is connected to an interior point rather than to an adjacent boundary point, as illustrated in Figure 1, which shows the maximum and minimum number of indentations for the same point set. If there are more interior points than boundary points, then there can be as many indentations as there are boundary points (i.e. an indentation may occur between each adjacent boundary point). If there are fewer interior points than boundary points, then there can be as many indentations as there are interior points (i.e. each interior point may be connected between two adjacent boundary points). In either case, the minimum number of indentations is one (provided that there are at least three boundary points and one interior point).

- volume 5, no. 1 (Fall 2012) 

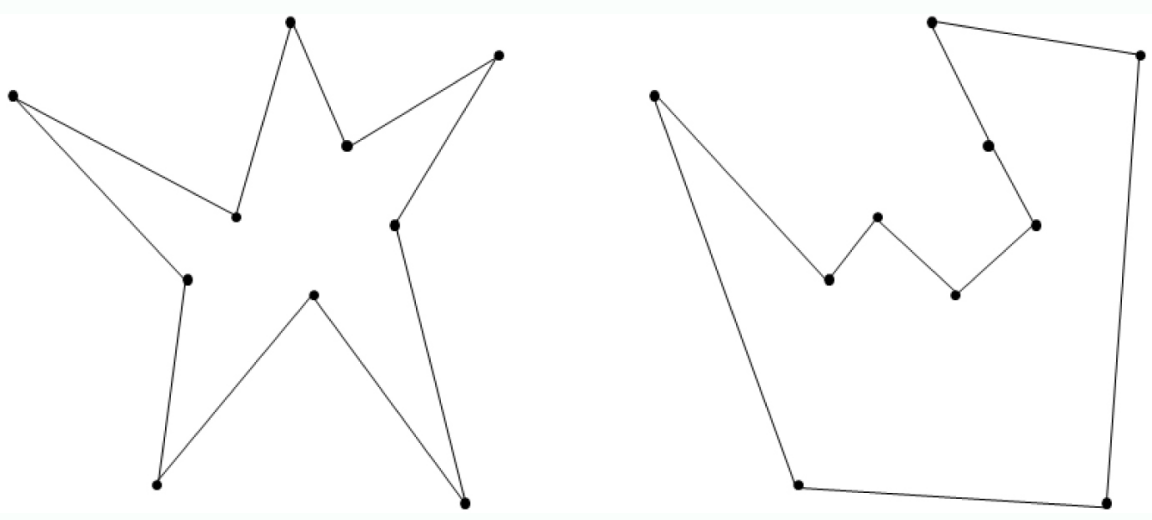

Figure 1.TSP tours showing maximum (left) and minimum (right) indentations.

MacGregor and Ormerod (1996) hypothesized that human solutions are guided by global properties of the convex hull, and that there may be preferences not only to connect boundary points in sequence but to connect them to each other. The latter preference would result in few indentations, which they tested by comparing the observed number of indentations with the chance distribution. The results of two experiments supported the hypothesis of few indentations. However, van Rooij et al. (2003) proposed an alternative interpretation of the findings. They suggested that, because participants tended to find near optimal tours, and because the optimal tours happened to have few indentations, then the results could have demonstrated a preference for optimal tours rather than for few indentations. Also, they considered it unlikely that the result would be replicated with random point sets. Subsequently, van Rooij, Schactman, Kadlec and Stege (2006) used randomly-generated instances and compared the number of indentations in participants' tours with the number in the corresponding optimal tours. Contrary to expectation, they concluded that there was a tendency to produce tours with fewer indentations than the optimal.

It may be noted that the alternative explanation offered by van Rooij et al. (2003) rests on two assumptions - that participants' tours are close to optimal, and that if optimal tours have few indentations, close to optimal tours will also have few indentations. However, even when the former assumption is met, the latter may not be. In fact, it is possible to produce almost identical point sets with almost identical optimal tours but with different numbers of indentations. This fact is used in generating the stimuli for Experiments 1 and 2, below, which were designed to test the "few indentations" hypothesis under more stringent conditions than in previous studies. In Experiment 1, participants generated solutions to five sets of TSP tasks, each set consisting of three variants. In one variant, the optimal tour had more indentations, in the second, fewer indentations, while in the third, both optimal tours were available. In the second experiment, participants were presented with pairs of optimal tours where one member of a pair had more indentations than the other. Participants were asked to judge which member of a pair had the shorter tour. 
A second overlooked observation from the earlier literature has to do with the location of the starting point that a solver chooses. Underlying the "few indentations" hypothesis of MacGregor and Ormerod (1996) was the implicit assumption that few indentations arise because participants work inwards from the convex hull or, in other words, that people choose to start from a point on the boundary. Subsequently, MacGregor et al. (2000) proposed the sequential convex hull model to explain human performance, and noted that the model provided a much better fit in cases where tours started from a point on the hull than from an interior point. They also reported that people showed a preference for starting on the hull. Based on the results of 48-node tours produced by 99 participants, $72 \%$ of observed tours started from a hull point, compared with $29 \%$ expected by chance. However, although the selection of a starting location may have theoretical implications for models of human performance, the observation does not appear to have been pursued. The present Experiment 1 provided an opportunity to examine whether there is a preference to start from the boundary, when hull and interior starting points are equally available.

Although the present experiments were not designed to critically test between theories, the results may have theoretical implications. To appreciate these, it may be useful to briefly summarize the main theoretical perspectives. A number of theories have now been proposed (Best, 2005; Kong \& Schunn, 2007; MacGregor et al., 2000; Pizlo, et al., 2006; van Rooij et al., 2003; Vickers et al., 2003), and a recent review identified a number of dimensions along which the theoretical accounts differ (MacGregor \& Chu, 2011). Important distinguishing features of models include: the direction of processing-global-to-local versus local-to-global (Vickers et al., 2003); clustering (e.g. Best, 2005; Pizlo et al., 2006); and the formation of a guiding contour (e.g. Kong \& Schunn, 2007; MacGregor et al., 2000). Table 1 summarizes the main features of the approaches in terms of these differences.

The majority of models propose clustering and global-to-local processing, but these features are not universal. Also, different mechanisms are proposed to underlie global-tolocal processing, including clustering, a guiding contour, or both. Finally, those models

\begin{tabular}{|l|l|l|l|l|}
\hline Model source & Local/Global & Cluster & Guiding Contour & Basis of Global Process \\
\hline Best & Global-local & Yes & Yes: convex hull & $\begin{array}{l}\text { Guiding contour/ } \\
\text { cluster }\end{array}$ \\
\hline Kong\&Schunn & Global-local & Yes & Yes: spline curve & $\begin{array}{l}\text { Guiding contour/ } \\
\text { cluster }\end{array}$ \\
\hline MacGregor et al. & Global-local & No & Yes: convex hull & Guiding contour \\
\hline Pizlo et al. & Global-local & Yes & No & Cluster \\
\hline Van Rooij et al. & Unspecified & No & No & NA \\
\hline Vickers et al. & Local-global & Yes & No & NA \\
\hline
\end{tabular}

Table 1. Characteristics of models of TSP problem solving.

- volume 5, no. 1 (Fall 2012) 
that propose a guiding contour differ in how it is implemented, as illustrated in Figure 2. As previously mentioned, the MacGregor et al. model (2000) proposes that solutions are based initially on the convex hull around the nodes on the boundary, which is modified as nodes are incorporated into the emerging path. In Best's model, the guiding contour starts with the convex hull through the centroids of clusters on the boundary, and adapts as interior clusters are incorporated. In Kong and Schunn's model, the guiding contour is a spline curve which passes through the centroids of all clusters, and remains stable throughout the solution process. The differences in guiding contours are illustrated in Figure 2. It is to these three models that the issues of indentations and starting points appear to be most immediately relevant.
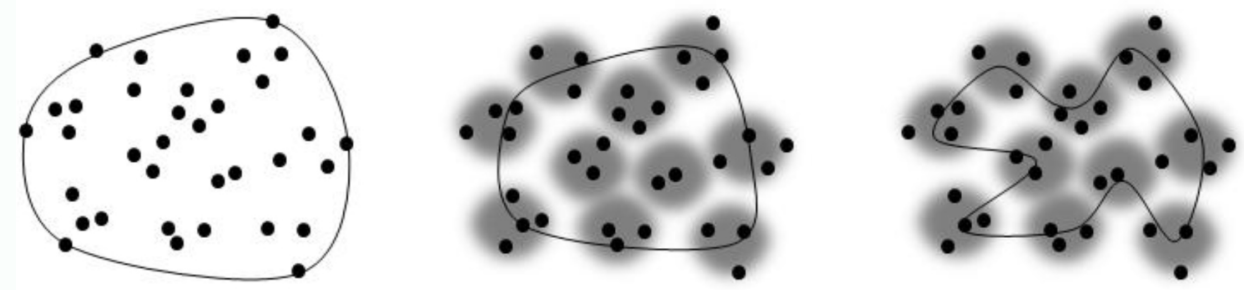

Figure 2. Guiding contours as proposed by three different models of TSP solving: MacGregor et al., 2000 (left), Best, 2005 (center), Kong \& Schunn, 2007 (right).

The sequential convex hull approach proposes that the first approximation to a tour is the convex hull-the set of arcs between each adjacent point on the boundary. Given a starting point on the hull and a direction of "travel," the model next considers whether to connect an interior point between the two end nodes of the current arc, on the basis of proximity. If no interior point is closer to the current arc than it is another arc on the convex hull, then the current arc is left intact and the process moves along the hull to the next arc. Other things being equal, the process therefore has a tendency to produce relatively convex tours - that is, tours with few indentations. The assumption is also that the typical process starts from a point on the boundary, and therefore would predict that a solver free to choose will select a starting point on the hull.

Best's (2005) model also assumes that a convex hull provides an initial guide to tour formation. However, the assumption is that the guiding contour passes through the centroids of clusters of nodes, rather than through nodes directly on the geometric boundary. Because of this, the process of tour construction will have to make a number of zigzags to incorporate points within clusters that lie on opposite sides of the guiding contour. Whenever such an oscillation returns to a point on the hull an indentation is formed. Thus, although the tours produced by the model will tend to be generally convex, the process may also produce a greater number of shallow indentations than those produced by the sequential convex hull process. Also, to the extent that the guiding contour influences the 
choice of a starting-point, then the model would predict a preference for starting-points close to, but not necessarily on, the geometric boundary of the point set.

In the model proposed by Kong and Schunn (2007) the guiding contour is a spline curve passing through the centroids of k-means clusters of points. Provided that there is at least one interior cluster, the guiding contour itself will contain at least one indentation, and potentially more. In addition, as with Best's model, oscillations may occur when points are connected that lie on opposite sides of the guiding contour. This will result in additional indentations if one end of the connection is a boundary point. The model therefore has the potential to produce the least convex tours of the three guiding contour models. In addition, assuming a starting-point is selected that is on, or close to, the spline curve (there may be no points on the curve), then the start may be virtually anywhere in the point set, depending on the specific form of the spline curve.

\section{Experiment 1}

The main purpose of the first experiment was to examine whether participants produce tours with fewer rather than more indentations, while controlling for effects of the number of indentations in the optimal tour. This was achieved by addressing two questions: first, do participants' tours tend to have fewer indentations than the corresponding optimal and, second, in cases where two (near) optimal solutions are simultaneously available, do participants tend to produce the one with fewer indentations?

Generating tours to answer the second of the two questions was made possible using a suite of programs designed under the direction of Zygmunt Pizlo. One feature of the application is that it can display the optimal tour to a point set while the location of a point is being changed by "dragging" it across the screen. When this occurs, the optimal tour may change in length but remain topologically equivalent across a range of positional translations of a single node until, at some point, a transformation may occur, in which the "shape" of the optimal tour changes. The transformation may include a shift in the number of indentations. This feature made it possible to identify two almost identical point sets that had optimal tours of the same, or virtually the same, lengths but a different number of indentations. A secondary purpose was to examine whether participants show a preference for selecting starting points from the boundary rather than the interior of a point set.

\section{Method}

\section{Participants}

Participants were 20 volunteers recruited from the campus community at the University of Victoria. 


\section{Stimuli}

The stimuli were fifteen TSP problems. Five had optimal solutions that had few indentations (forming the Low Indent condition), 5 had optimal solutions with more indentations (High Indent condition) and 5 were designed so that either a high indent or low indent optimal solution was possible (High/Low Indent condition).

The stimuli were produced in the following way. Using the software described above, 10-node point sets were randomly generated until one was obtained which had 5 boundary points and 5 interior points. The set was constrained to occupy a display of $512 \times 512$ pixels, and points had a radius of 8 pixels. Next, the most central point, $x$, was identified, and moved either vertically or horizontally in the direction of the furthest boundary. Movement continued until the optimal solution changed from Optimal A to Optimal B, having more or fewer indentations, at location $a b$, identified as the "indifference location". (In some cases, the "indifference location" was two locations, a pixel or two apart, ab, associated with Optimal A and $a b^{*}$, associated with Optimal B. In such cases solutions A and $B$ were either the same length or differed by no more than $0.25 \%$.) Then point $x$ continued to be moved in the same direction until either solution B transformed or a boundary was reached. Location $b$ was identified as halfway between this point and location ab. Next, point $x$ was moved back in the direction of location $a b$, where the optimal changed back to optimal tour A. Movement continued through $a b$ on the same trajectory until tour $A$ changed to an optimal with another shape or a boundary was reached. Halfway between this location and $a b$ was identified as location $a$. In this way, three locations were identified for point $x$-location $a$, associated with optimal tour A only, location $a b$, associated with optimal tour A and optimal tour B, and location $b$, associated with optimal tour B only. Three different point sets were thus formed from the original random set, differing only in the location of $x$. One of the three had an optimal solution with fewer indentations, one with more, and the third had two optimal (or virtually optimal) solutions, one with fewer, one with more.

Figure 3 illustrates the results of this process for one stimulus set. The original random point set is shown in the top left panel and the associated optimal tour is shown in the panel immediately below. In this case the optimal had two indentations. The next panel to the right in the upper row shows the point set after the most central node had been moved vertically downwards through a short distance. The optimal, shown in the lower panel, was topologically equivalent to the first, with two indentations. The third panel in the first row contains a point set identical, or virtually identical, to the previous point set, where an alternative topologically different optimal tour was available. This alternative optimal is shown in the panel immediately below, and had three indentations. The final

panel shows the point set with the central node moved further down to a position where the three indent optimal tour was stable. 


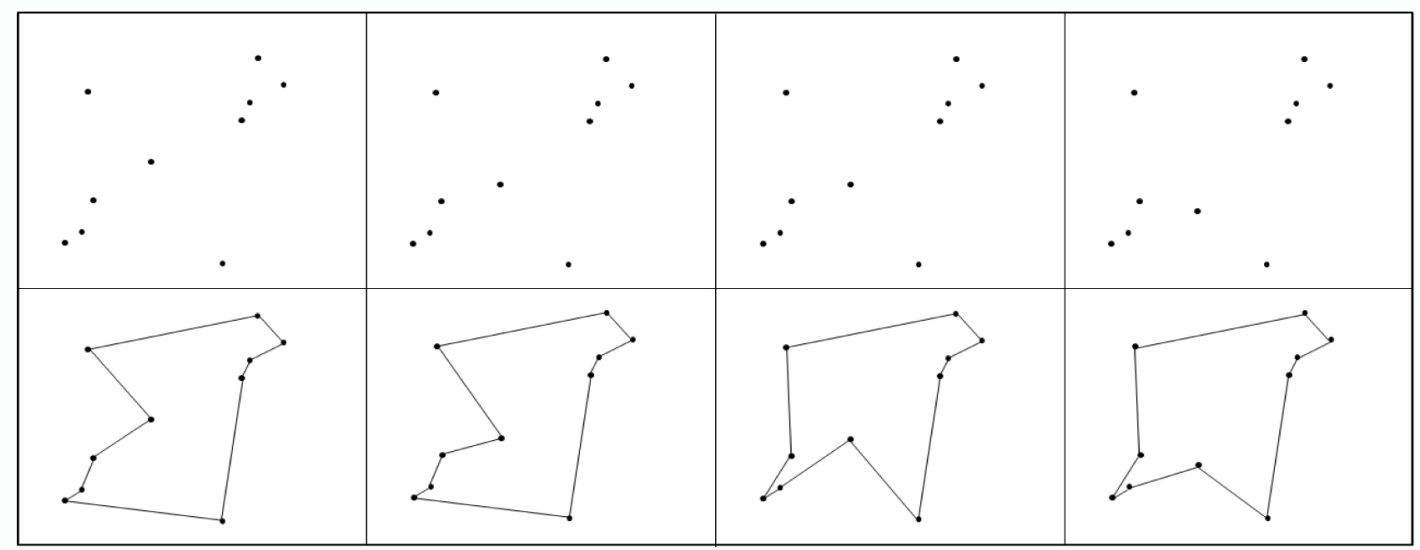

Figure 3. Sample stimulus set from Experiment 1. The top row shows four related point sets and the bottom row shows the associated optimal paths.

This procedure was repeated until there were five independent sets each consisting of three related point sets-one yielding an optimal solution with fewer indents, one an optimal with more indents, and the third having both optimal tours available. The number of indentations for optimal solutions in the five stimulus sets were1, 2, 2, 2, and 3 for the Low Indent condition (mean $=2$ ) and 2, 3, 3, 3, and 4 respectively for the High Indent condition (mean = 3). For the five point sets in the High/Low Condition each of two optimal tours were available, and in each case the two were of identical length or differed by no more than $0.25 \%$, or approximately 2 pixels, on average, over a mean optimal path length of 1550 pixels.

\section{Procedure}

Participants were tested individually and each completed all fifteen stimulus sets. The order of presentation of stimuli was randomized, subject to the constraint that no member of the same set was presented immediately after another. Also, the second in order of each set of three was rotated through $180^{\circ}$, to help reduce the chance that a participant might notice similarities among related point sets. Stimuli were presented on screen and participants indicated their paths by pointing-and-clicking.

\section{Results and discussion}

The 20 participants produced a total of 300 tours. Twenty-three tours (8\%) had crossed arcs that made the visual identification of indentations ambiguous, and these tours were excluded from further analysis. For the remaining 277 tours the number of indentations in each tour was assessed. During this process, it emerged that a point set in the Low Indent condition qualified for the High/Low condition, because a second "optimal" tour was produced that was both within $0.25 \%$ of the optimal and had a different number of indentations from the optimal. The results for this point set were therefore included in the High/Low condition. 


\section{Number of indentations}

The first analysis compared the number of observed indentations with the number of indentations in the corresponding optimal tours, the comparison recommended by van Rooij et al. (2006). For the 277 participant tours, the number of indentations ranged from 1 to 4 , with a mean of 2.41. To test significance, the number of indentations in the optimal tour was subtracted from the observed number for each of the 277 tours. For convenience, the resulting value will be referred to as the relative number of indentations. (For tours in the High/Low condition, the average of the two optimal solutions was used so that, for example, if one optimal had 2 indentations and the other 3 , a value of 2.5 was used.) A relative score of zero would support the null hypothesis, that participants produce indentations corresponding to the optimal tour, while a relative score of less than zero would reflect a preference for minimizing indentations beyond the requirements of the optimal tour. A mean relative number of indentations score was found for each participant, and the average of these was -0.13 (standard error $=0.05$ ), which was significantly different from $0, t(19)=-2.44, p<.01$. The direction of the difference indicated that participants produced tours with significantly fewer indentations than the optimal tours.

The fact that participants produced tours with fewer indentations than the optimal lends support to the hypothesis of a preference for fewer indentations. The next analysis put the hypothesis to a more stringent test, by examining only the tours from the High/ Low condition that conformed to one of the two (close to) optimal solutions. This allowed a test of whether tours with fewer indentations are preferred when controlling for potential effects of optimality.

Of the 112 tours produced in the High/Low condition, $60(54 \%)$ were optimal. Of these optimal solutions, 41 (68\%) were the alternative with fewer indentations. Significance was tested as in the previous analysis but, because one participant had no optimal tours, $\mathrm{n}$ dropped to 19 . The mean relative number of indentations was -0.18 (standard error $=0.08$ ), $t(18)=-2.26, p<.05$. Thus, when two virtually optimal solutions were available, the one with fewer indentations was produced significantly more often than the alternative.

\section{Starting point preferences}

For each participant the mean number of the 15 tours that started from a boundary point was found. The overall mean of these was $10.7(71 \%)$, compared with an expected mean of 7.5 if starting points were selected randomly. The difference was significant by a one-sample t-test, which indicated a preference to start a tour from the boundary, $t(19)=3.19, p<.01$. For the remaining tours, starting from interior locations, $51(18 \%)$ began from the point closest either to the centroid of the convex hull or the centre of mass of the point set (or both), 22 (8\%) started from a point nearer the centre than the boundary, and $10(4 \%)$ started from a point closer to, but not on, the boundary. Thus, tours that did not start from a boundary point appeared to exhibit a preference for a central location. 
Although there was an overall preference to start tours from a boundary point, this tendency appeared to vary across individuals. The frequency of hull starts ranged across participants from a low of 7\% (i.e. 1 of 15 tours started on the hull) to a high of $100 \%$, with a mean of $71 \%$. The observed distribution differed significantly from chance, $v^{2}(19)=35.04, p=.01$, indicating systematic individual differences in starting point preferences.

\section{Results and discussion}

The primary result of Experiment 1 was the finding that participants produced tours with fewer indentations than the optimal. Further, this tendency persisted with point sets that had two optimal solutions, where participants showed a significant tendency to produce the optimal with the fewer indentations of the two. A secondary finding suggested an additional factor that may be involved in this preference, in that starting points on the boundary were selected more frequently than in the interior, by a substantial margin. However, the question remains as to whether or not starting preferences and number of indentations are causally linked and, if so, in what sequence. One possibility is that there is a preference for selecting boundary starting points, and that starting from a boundary point leads to fewer indentations. A quite different possibility is that there is a preference for fewer indentations, and boundary starting points are perceived as being more likely to achieve this end. The latter possibility implies that minimizing indentations may be a strategic choice that exerts a guiding influence on tour construction. If this were the case, then we would expect that the preference might be cognitively available more generally as, for example, in judging the quality of tours already produced. Experiment 2 was conducted to address this issue.

\section{Experiment 2}

Experiment 2 investigated whether the number of indentations in a completed tour influences the perception or judgment of its length. If it does, and if the perception is that fewer indentations are associated with shorter-looking tours, this perception may influence the process of tour construction to produce tours that have few indentations. If it does not, then it seems less likely that an overt strategy of minimizing indentations plays a role in tour construction.

The experimental approach was similar to the one introduced by Ormerod and Chronicle (1999) except that, where they asked participants whether a single tour was optimal or non-optimal, the present experiment asked participants to indicate which of a pair of tours was the shorter.

- volume 5, no. 1 (Fall 2012) 


\section{Method}

\section{Participants.}

Participants were the 20 volunteers who took part in Experiment 1.

\section{Stimuli}

The stimuli were 10 pairs of TSP instances, each with the optimal tour displayed. Five pairs consisted of 15 nodes and five of 20. Pairs were generated in a manner similar to the previous experiment. That is, a point set was randomly generated and then the most central point was moved until the optimal changed to one with more or fewer indentations. The point sets immediately before and immediately after this shift constituted each member of a pair. For the 15-node point sets, the procedure was repeated until 5 different pairs were obtained where the number of indentations between each member of a pair differed by two. For the 20-node point sets, 5 pairs were obtained where each member of a pair differed by one indentation. The stimuli were printed in a booklet with each pair on a single page. One member of each pair was selected randomly to be rotated through $90^{\circ}$, to disguise the fact that the tours were generated using virtually identical point sets. An example of a stimulus pair is shown in Figure 4.

\section{Procedure}

The participants from Experiment 1 took part in the present experiment, immediately following Experiment 1. The test booklet was provided and participants were asked to indicate which member of each pair had the shorter path. The instructions included the statement "In doing the task, don't try to measure or estimate the length, but be guided by your overall perception of which seems the shorter of the two. The entire task should require only a few minutes to complete."
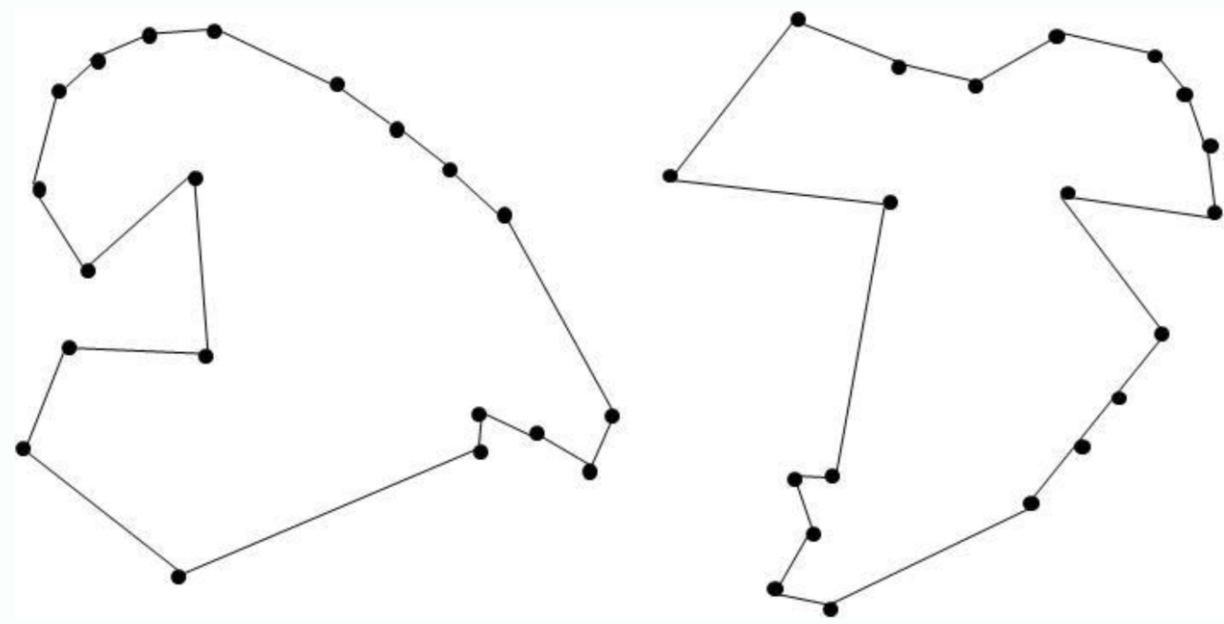

Figure 4. Sample stimulus pair showing two optimal solutions with different numbers of indentations The point set on the left has been rotated clockwise through $90^{\circ}$ to create the point set on the right. 


\section{Results and Discussion}

The result of interest was whether tours with fewer indentations tended to be judged as shorter than their matched counterparts with more indentations. The average proportion of times each participant selected the option with fewer indentations was calculated, and found to range from 0.1 to 0.9 , with a mean of $0.55(s=0.25)$. The difference from the expected proportion of 0.50 did not approach significance, by a one-sample t-test, $t(19)=0.95, p=.36$ ). The results indicated that, on average, participants did not use number of indentations as a basis for comparing tour lengths.

In the above analysis, repeated observations on the same individuals were treated as dependent, following the usual assumption that, because of individual differences, repeated observations will not be independent. A more formal criterion is that two observations are independent if the conditional probability of one, given the other, is the same as the unconditional probability (Kenny \& Judd, 1986), and a potential issue is that repeated measures in some TSP tasks may meet the definition of independence. For example, prior research with TSPs having few nodes (20 or fewer) has reported the absence of reliable individual differences across repeated trials (MacGregor \& Ormerod, 1996). In such cases an individual's performance on one TSP is no more predictive of subsequent performance than the performance of an unrelated individual, which meets the definition of independence of observations. If independent observations are treated as dependent, statistical power is reduced and the probability increases of failing to reject the null hypothesis when it is false. To check for this possibility in the present case, the results were tested for independence of observations, using a liberal criterion of $p=.20$, two-tailed, as suggested in Cook and Kenny (2005). To do so, the number of times each individual selected the fewer indents option as shorter was compared with the number expected by chance, resulting in $\mathrm{v}^{2}(19)=21.83, p=.29$. The result failed to reach the recommended criterion of $p<.20$ to reject the null hypothesis of independence. Based on this, the data were reanalyzed, treating all 197 observations as independent. Using a one-sample z-test, the results indicated that the proportion of observations in which the fewer indentation tour was judged as shorter did not differ from chance, $z=0.50$, $p=.62$. Thus, whether the observations are treated as dependent or independent, there is no evidence that the number of indentations influences judgment of tour length. Given that people do not appear to perceive fewer indentations as associated with shorter paths, nor are there reliable individual differences in any tendency to do so, it seems

unlikely that individuals would adopt a strategy of minimizing indentations as a guide for constructing optimal tours.

- volume 5, no. 1 (Fall 2012) 


\section{General Discussion}

The results of the experiments support several conclusions. First, human solutions to the present small TSPs tended to have few indentations, even after controlling for the potential effects of the number of indentations in the optimal tour. Second, there was no evidence of individual differences in this tendency. Third, producing tours with few indentations does not appear to result from a strategic choice, in that participants did not equate fewer indentations with shorter tours in Experiment 2. It therefore seems more likely that few indentations are an outcome rather than a goal of the process of tour production. Fourth, starting points on the boundary were preferred over any other location, with $71 \%$ of tours starting from a point on the hull, almost identical to the figure of $72 \%$ reported by MacGregor et al. (2000). The implications of these results for theories of tour production are next considered.

The third finding, that few indentations are more likely to be a product than a strategy of tour construction, does not discriminate between theories, for the simple reason that no theory proposes such a strategy. Nevertheless, one theoretical approach, the crossingavoidance hypothesis, is based on strategic choice, so the idea that tours may be guided by goals or sub-goals other than minimizing length is not unknown. However, to the extent that all models produce completed tours, then the resulting tours will have indentations as a by-product of the construction process (provided a point set has at least one interior point). The more pertinent question is whether the processes are likely to limit the number of indentations. As discussed in the introduction, of the models proposing that tours are built from a guiding contour, it appears that the sequential convex hull model is likely to produce fewer indentations than the approaches proposed by Best (2005) or Kong and Schunn (2007). As far as they go, the present results would therefore appear to support the former over the latter.

If the assumption is made that a start point will be selected that lies on (or as close as possible to) the guiding contour, then the fourth finding, that $71 \%$ of starting points selected by participants were located on the boundary, also appears to support the sequential convex hull model over the alternative guiding contour based theories. At the same time, the fact that $18 \%$ of starts favoured the most central point is not consistent with the sequential convex hull model, and indicates that other systematic preferences may also be at play. If clustering approaches assume that a point nearest the centre of a cluster is selected as the start, then a possible explanation is provided by the pyramid model (Pizlo et al., 2006). The model adopts a top down hierarchical clustering approach, so that the stimulus representation at the highest level is a single cluster, the centre of which would correspond to the centre of mass of all the points. The point closest to the centre of the point set would therefore provide the initial starting location. In addition, the finding of individual differences in starting point selection suggests that more than 
one heuristic may be required to account for TSP performance and preferences, consistent with the conclusions of MacGregor, Chronicle, and Ormerod (2006) and of Vickers, Lee, Dry, Hughes, and McMahon (2006). However, it is also possible that starting points are selected because of non-TSP specific factors such as perceptual saliency, which could explain the preferences for both boundary and central locations. Further research will be necessary to isolate the relevant factors.

Because the research was designed to test the hypothesis of limited indentations, and not to critically test between theories, these potential implications are speculative. Additional limitations to the findings arise from the small size of TSPs used and the fact that starting selections were observed rather than being experimentally manipulated. Nevertheless, within these limits, the research found systematic response patterns concerning indentations and starting points that may challenge many current theories for an explanation.

\section{Acknowledgments}

The author wishes to thank Zygmund Pizlo, Zheng Li, Emil Stefanov, and Jack Saalweachter for making available the computer program that made the present research possible. The research was partially supported by an NSERC grant from the Government of Canada.

\section{References}

Basso, D., Lotze, M., Vitale, L., Ferrer, F., Bisiacchi, P., Olivetti Belardinelli, M., Rossini, P. M., \& Birbaumer, N. (2006). The role of prefrontal cortex on visuo-spatial planning: A repetitiveTMS study. Experimental Brain Research, 171, 411-415. http://dx.doi.org/10.1007/ s00221-006-0457-z

Best, B. J. (2005). A model of fast human performance on a computationally hard problem. Proceedings of the 27th Annual Conference of the Cognitive Science Society, 256-261.

Cohen, J. (1962). The statistical power of abnormal-social psychological research: A review. Journal of Abnormal and Social Psychology, 65, 145-153. http://dx.doi.org/10.1037/ h0045186

Cook, W. L., \& Kenny, D. A. (2005). The actor-partner interdependence model: A model of bidirectional effects in developmental studies. International Journal of Behavioral Development, 29(2), 101-109. http://dx.doi.org/10.1080/01650250444000405

Dry, M., Lee, M. D., Vickers, D., \& Hughes, P. (2006). Human performance on visually presented traveling salesperson problems with varying numbers of nodes. Journal of Problem Solving, 1(1), 20-32.

Findlay, J. M., \& Brown, V. (2006). Eye scanning of multi-element displays: I. Scanpath planning. Vision Research, 46, 179-195. http://dx.doi.org/10.1016/j.visres.2005.06.010

- volume 5, no. 1 (Fall 2012) 
Gibson, B. M., Wasserman, E. A., \& Kamil, A. C. (2007). Pigeons and people select efficient routes when solving a one-way "traveling salesperson" task. Journal of Experimental Psychology: Animal Behavior Processes, 33, 244-261. http://dx.doi.org/10.1037/00977403.33.3.244

Graham, S. M., Joshi, A., \& Pizlo, Z. (2000). The traveling salesman problem: A hierarchical model. Memory \& Cognition, 28, 1191-1204. http://dx.doi.org/10.3758/BF03211820

Kenny, D. A., \& Judd, C. M. (1986). Consequences of violating the independence assumption in analysis of variance. Psychological Bulletin, 99, 422-431. http://dx.doi. org/10.1037/0033-2909.99.3.422

Kong, X., \& Schunn, C. D. (2007). Global vs. local information processing in visual/spatial problem solving: The case of traveling salesman problem. Cognitive Systems Research, 8(3), 192-207. http://dx.doi.org/10.1016/j.cogsys.2007.06.002

Kong, X., Schunn, C. D., \& Wallstrom, G. L. (2010). High regularities in eye-movement patterns reveal the dynamics of the visual working memory allocation mechanism. Cognitive Science, 34, 322-337. http://dx.doi.org/10.1111/j.1551-6709.2009.01075.x

MacGregor, J. N., Chronicle, E. P., \& Ormerod, T. C. (2006). A comparison of heuristic and human performance on open versions of the traveling salesperson problem. Journal of Problem Solving, 1(1), 33-43.

MacGregor, J. N., \& Chu, Y. (2011). Human Performance on the Traveling Salesman and Related Problems: A Review. Journal of Problem Solving, 3(2), 1-29.

MacGregor, J. N. \& Ormerod, T. (1996). Human performance on the traveling salesman problem. Perception \& Psychophysics, 58, 527-539. http://dx.doi.org/10.3758/BF03213088

MacGregor, J. N., Ormerod, T. C., \& Chronicle, E. P. (2000). A model of human performance on the traveling salesperson problem. Memory and Cognition, 28, 1183-1190. http:// dx.doi.org/10.3758/BF03211819

Ormerod, T. C. \& Chronicle, E. P. (1999). Global perceptual processing in problem-solving: The case of the traveling salesperson. Attention, Perception and Psychophysics, 61, 1227-1238. http://dx.doi.org/10.3758/BF03207625

Pizlo, Z., Stefanov, E., Saalweachter, J., Haxhimusa, Y., \& Kropatsch, W. G. (2006). Traveling salesman problem: A foveating pyramid model. Journal of Problem Solving, 1(1), 83-101.

Ragni, M., \& Löffler, C. M. (2010). Complex problem solving: Another test case? Cognitive Processes, 11, 159-170. http://dx.doi.org/10.1007/s10339-009-0344-1

van Rooij, I., Schactman, A., Kadlec, H., \& Stege, U. (2006). Perceptual or analytical processing? Evidence from children's and adult's performance on the Euclidean traveling dalesperson problem. Journal of Problem Solving, 1(1), 44-73.

van Rooij, I., Stege, U., \& Schactman, A. (2003). Convex hull and tour crossings in the Euclidean traveling salesperson problem: Implications for human performance studies. Memory and Cognition, 31, 215-220. http://dx.doi.org/10.3758/BF03194380 
Vickers, D., Butavicius, M. Lee, M., \& Medvedev, A. (2001). Human performance on visually presented traveling salesman problems. Psychological Research, 65, 34-45. http:// dx.doi.org/10.1007/s004260000031

Vickers, D., Lee, M. D., Dry, M., \& Hughes, P. (2003). The roles of the convex hull and number of intersections in performance on visually presented traveling salesperson problems. Memory \& Cognition, 31, 1094-1104. http://dx.doi.org/10.3758/BF03196130

Vickers, D., Lee, M. D., Dry, M., Hughes, P., \& McMahon, J. A. (2006). The aesthetic appeal of minimal structures: Judging the attractiveness of solutions to traveling salesperson problems. Perception \& Psychophysics, 68, 32-42. http://dx.doi.org/10.3758/ BF03193653.

- volume 5, no. 1 (Fall 2012) 